\title{
Spezies-abhängige In-vitro-Empfindlichkeit von Malassezia gegenüber Antimykotika*
}

\section{Species Specific Susceptibility of Malassezia against Antifungal Agents in vitro}

Autoren

Institute

\section{P. Nenoff ${ }^{1,2}$, A. Kalinowska-Pujdak ${ }^{1 * *}$, A. Schmalreck ${ }^{3}$}

Klinik für Dermatologie, Venerologie und Allergologie, Universitätsklinikum Leipzig

Laboratorium für medizinische Mikrobiologie, Mölbis

Mikrobiologische Beratung \& Service, München

\section{Bibliografie}

Dol $10.1055 / \mathrm{s}-2006-944857$

Akt Dermatol 2007; 33;

26-32 @ Georg Thieme

Verlag KG Stuttgart · New York ISSN 0340-2541

Korrespondenzadresse

Prof. Dr. med. Pietro Nenoff Laboratorium für medizinische Mikrobiologie Straße des Friedens 8 04579 Mölbis

Tel.: 034347-50 323

Fax: 034347-50 123

info@mykologie-experten.de

\section{Zusammenfassung}

$\nabla$

Lokal und systemisch applizierbare Antimykotika (Fluconazol, Ketoconazol, Voriconazol, Itraconazol, 5-Flucytosin und Amphotericin B) wurden hinsichtlich ihrer In-vitro-Aktivität gegenüber Malassezia getestet. Zum Einsatz kamen insgesamt 81 Malassezia-Stämme (Patientenisolate sowie Referenzstämme), die aufgrund konventioneller biochemischer Differenzierung sowie mittels Fourier-Transform-Infrarot-Spektroskopie (FT-IRS) verschiedenen Spezies zugeordnet werden konnten.

Die Ermittlung der minimalen Hemmkonzentrationen (MHK) basierte auf einem selbst entwickelten Mikrodilutionstest. Dazu wurden rehydratisierte Antimykotika mit standardisierten Hefezellsuspensionen aufgelöst.

\section{Einleitung}

Malassezia (M.) ist ein lipophiler Spross- oder Hefepilz, der zur normalen menschlichen Hautflora gerechnet wird [1-3]. Es handelt sich um einen fakultativ-pathogenen Erreger, welcher die Fähigkeit besitzt, unter bestimmten Bedingungen Ursache einer Reihe dermatologischer Erkrankungen zu werden [4-6]. Dazu zählen Pityriasis versicolor, M.-Follikulitis, seborrhoisches Ekzem und Kopfschuppen [7-11]. In den letzten Jahren wurden aufgrund morphologischer, physiologischer, ultrastruktureller und vor allem molekularbiologischer Untersuchungen neue Taxa den drei bereits bekannten Arten M. furfur, M. pachydermatis und $M$. sympodialis hinzugefügt. Diese

\footnotetext{
* Herrn Prof. med. Uwe-Frithjof Haustein zum 70. Geburtstag gewidmet.

** Aktuelle Adresse: Mathias-Hospital, Kardiologische
} Klinik, Frankenburgstraße 31, 48431 Rheine
Die MHK-Werte der einzelnen Malassezia-Spezies variierten innerhalb eines Antimykotikums. Der Vergleich zwischen den eingesetzten Wirkstoffen erbrachte signifikante Unterschiede. Wie erwartet, hemmten die Azolantimykotika (Fluconazol, Ketoconazol, Voriconazol und Itraconazol) das Wachstum in vitro schon bei niedrigen Konzentrationen. Voriconazol, Itraconazol und Ketoconazol hemmten das Wachstum in vitro am stärksten, was mit anderen Studien übereinstimmt. Offensichtlich gibt es Unterschiede der In-vitro-Empfindlichkeit der verschiedenen $\mathrm{Ma}$ lassezia-Spezies. Malassezia furfur ist am wenigsten empfindlich gegenüber mehreren Antimykotika, dagegen sind Malassezia sympodialis, Malassezia globosa und Malassezia obtusa deutlich empfindlicher, d.h. sie wiesen niedrigere MHKWerte auf.

umfassen $M$. globosa, $M$. obtusa, $M$. restricta, $M$. slooffiae, M. dermatis sowie M. japonica [12 - 16]. Die Bestimmung der minimalen Hemmkonzentration (MHK) der Azolantimykotika und anderer antimykotisch wirkender Substanzen gegenüber Sprosspilzen ist durchaus problematisch. Es gibt einen gebilligten Standard des National Committee for Clinical Laboratory Standards (NCCLS), der auf einer Bouillonverdünnungstechnik basiert. Demgegenüber nutzt die vom Deutschen Institut für Standardisierung (DIN) empfohlene Mikrodilutionsmethode das High-resolution-Medium für Sprosspilze der Gattung Candida. Beide Methoden wurden jedoch für schnell wachsende Candida-Arten, allenfalls auch für die etwas langsamer wachsende klinisch-relevante Spezies Cryptococcus neoformans, entwickelt. Die Empfindlichkeitstestung von Malassazia spp. ist aufgrund der Lipophilie dieser Hefepilze sowie des deutlich langsameren Wachstums mit diesen Methoden nicht möglich. 
Beschrieben ist die MHK-Bestimmung für Malassezia mittels Agardilutionstest unter Verwendung von Diagnostic Sensitivity Test-Agar (D.S.T.-Agar, Oxoid), der 2\% Olivenöl und 0,2\% Tween 80 enthält [17,18], oder von Dixon-Agar mit Glyzerolmonostearat. Letzteres wurde von Gupta et al. [19] für die Testung von $M$. globosa, M. obtusa und M. restricta als wachstumstimulierendes Medium benutzt. Auf diese Weise konnten in der Vergangenheit Antimykotika und antimykotisch wirkende Substanzen, wie antiseborrhoische Mittel, australisches Teebaumöl, Steinkohlenteer und Dithranol, getestet werden.

Hier erfolgte die In-vitro-Empfindlichkeitstestung von Malassezia gegenüber lokal und systemisch applizierbaren Antimykotika (Fluconazol, Ketoconazol, Voriconazol, Itraconazol, 5-Flucytosin und Amphotericin B). Die Ermittlung der MHK-Werte basierte auf einem selbst entwickelten Mikrodilutionstest.

\section{Material und Methoden}

$\nabla$

\section{Malassezia-Stämme}

Im Rahmen der vorliegenden Studie wurden 81 MalasseziaStämme - 64 klinische Isolate von Patienten der Universitätshautklinik Leipzig - untersucht ( $\odot$ Tab. 1). Dazu kamen 17 Referenzstämme vom Centraalbureau voor Schimmelcultures (CBS, Utrecht, Niederlande) sowie der American Type Culture Collection (ATCC, Manassas, Virginia, USA). Die Merkmale dieser Reinkulturen unterscheiden sich nicht vom Ausgangsstamm dank limitierter Anzahl von Passagen. Mehrere als Referenzstämme verwendete Wildisolate wurden freundlicherweise von Herrn Prof. Dr. P. Mayser, Zentrum für Dermatologie und Andrologie, Justus Liebig Universität Gießen, zur Verfügung gestellt.

Die klinischen Stämme wurden aus Hautschuppen von Patienten gewonnen, bei denen $M$. spp. Ursache der zugrunde liegenden Dermatose war: Pityriasis versicolor, seborrhoisches Ekzem, und dessen milde Form, die Pityriasis simplex capillitii, also die vermehrte Kopfschuppung ( $\bullet$ Tab. 2).

\section{Kultivierung von Malassezia \\ $\nabla$}

Die primäre kulturelle Anzucht erfolgte bei $37^{\circ} \mathrm{C}$ auf Sabouraud 4\%-Glukose-Nährboden (Sifin, Berlin; pH 5,7), der Olivenöl und $0,2 \%$ Tween 80 enthielt. Jedes Isolat wurde nach siebentägiger Inkubationszeit auf Objektträger ausgestrichen, getrocknet, hitzefixiert und mit Methylenblau gefärbt und mikromorphologisch untersucht.

Die Sprosspilzisolate wurden auf mDixon-Agar [20] ausgestrichen. Dieses Medium hat den Vorteil, dass auf den Zusatz von Olivenöl und Tween 80 verzichtet werden kann. Als wachstumsförderndes Substrat ist Glyzerolmonostearat beigemengt. Die Inkubation erfolgte bei $37^{\circ} \mathrm{C}$. Jeder Stamm wurde gleichzeitig auf Sabouraud 4\%-Glukose-Medium ohne Zusatz von mehrkettigen Fettsäuren beimpft. Das Wachstum wurde mit dem auf dem fetthaltigen mDixon-Nährboden verglichen. Wenn das Isolat sowohl auf dem lipidhaltigen als auch auf dem lipidfreien Nährmedium in demselben Ausmaß wuchs, musste der untersuchte Stamm als nicht obligat-lipophil angesehen werden, es handelte sich dann um $M$. pachydermatis als einzige fakultativ-lipophile Spezies innerhalb der Gattung [21].
Tab. 1 Klinische Diagnosen und ihnen entsprechende Herkunftstellen

\begin{tabular}{llcll}
\hline Diagnose & \multicolumn{2}{l}{ Entnahmestelle } & Anzahl [n] \\
& Brust & Rücken & $\begin{array}{l}\text { Kopf- } \\
\text { schuppen }\end{array}$ & $\mathbf{n = 6 4}$ \\
$\begin{array}{l}\text { Pityriasis } \\
\text { versicolor }\end{array}$ & 2 & 14 & 0 & 16 \\
\hline $\begin{array}{l}\text { seborrho- } \\
\text { isches Ekzem }\end{array}$ & 7 & 12 & 0 & 19 \\
\hline $\begin{array}{l}\text { Kopf- } \\
\text { schuppen }\end{array}$ & 0 & 0 & 29 & 29 \\
\hline $\mathrm{n}=65$ & 9 & 26 & 29 & 64 \\
\hline
\end{tabular}

Tab. 2 Übersicht der 17 Malassezia-Referenzstämme (CBS - Centraalbureau voor Schimmelcultures, Utrecht, Niederlande). Die Wildstämme wurden freundlicherweise von Herrn Prof. Dr. Peter Mayser, Gießener Universitätshautklinik, zur Verfügung gestellt.

\begin{tabular}{|l|l|}
\hline $\begin{array}{l}\text { Spezies } \\
\text { M. furfur }\end{array}$ & Bezeichnung der Referenzstämme \\
& EG 658, CBS 6000, CBS 4162, CBS 6046, CBS \\
\hline M. sympodialis & $\begin{array}{l}\text { CBS 7979, ATCC 42 132, Wildstämme 18, 43, 48, } \\
49,80,70\end{array}$ \\
\hline M. obtusa & CBS 7968 \\
\hline M. globosa & Wildstämme 16, 26 \\
\hline * Bezeichnung nach der geografischen/lokalen Herkunft
\end{tabular}

\section{Differenzierung der Malassezia-Isolate}

Die Malassezia-Stämme wurden basierend auf der Verstoffwechselung von verschiedenen Substraten, u.a. der Assimilation von Polyoxyethylensorbitanestern (Tween 20, 40, 60, 80), der Katalase-Reaktion sowie L-Tryptophan- und Esculinspaltung, konventionell biochemisch identifiziert. Die Speziesdifferenzierung wurde für alle Stämme mittels Fourier-Transform-Infrarot-Spektroskopie bestätigt [22].

\section{In-vitro-Empfindlichkeitstestung}

$\nabla$

Die In-vitro-Empfindlichkeitsbestimmung wurde auf Mikrotitrationsplatten (Micronaut-SB, Merlin, Bornheim-Hersel) durchgeführt und beruht auf der Rehydratisierung von Antimykotika durch die Zugabe einer standardisierten Sprosszellsuspension. Die fertige Platte enthielt sechs Antimykotika in zwölf verschiedenen Konzentrationen. Zu den getesteten Antimykotika gehörten: 5-Flucytosin, Amphotericin B, Fluconazol, Itraconazol, Ketoconazol und Voriconazol. Wachstumskontrollen wurden jeweils mitgeführt.

Für die MHK-Bestimmung wurden folgende Medien eingesetzt. Wenn kein Wachstum zu verzeichnen war, wurde das nächste flüssige Medium verwendet:

a) HR (High-Resolution)-Medium, das sich für die MHK-

Bestimmung anderer Sprosspilze, z. B. Candida als geeignet erwiesen hat,

b) HR-Medium mit Tween 80

c) Dixon-Medium ohne Pepton

d) Minimalmedium $+2 \%$ Glukose + Olivenöl $2 \%$. 


\section{Eignung der Medien \\ $\nabla$}

Die Dixon-Platten wurden mit $1 \mathrm{ml}$ HR-Puffer abgeschwemmt und Verdünnungsreihen vorbereitet. In die Mikrotitrationsplatten mit vorgelegten Antimykotika kamen $100 \mu \mathrm{l}$ HR-Medium pro Vertiefung. Es wurden folgende Konzentrationen der Sprosspilzsuspensionen pro Vertiefung getestet: $10^{3}, 10^{4}, 10^{5}$ und $10^{6} \mathrm{KbE} / \mathrm{ml}$ (Kolonie-bildende Einheiten/ml). Das Wachstum wurde jeden Tag beurteilt.

Der Versuch mit HR-Medium und HR mit 0,1 \%iger Lipidsubstitution (in Form von Tween 80) scheiterte, da kein Wachstum in der Kontrollvertiefung nachgewiesen werden konnte. Mit der Dixonbouillon war Wachstum schon nach 24-48 Stunden zu beobachten, so dass dieses Medium für die MHK-Bestimmung als geeignet eingeschätzt wurde.

\section{Herstellung des Inokulums \\ $\nabla$}

Die sieben Tage alten Kolonien wurden mit $4 \mathrm{ml}$ Dixon-Bouillon abgeschwemmt (die Ausgangskeimzahl betrug $10^{7}$ bis $10^{8} \mathrm{KbE} /$ $\mathrm{ml}$ ), in Reagenzgläser übertragen und homogenisiert.

- Aus jedem Reagenzglas wurde $1 \mathrm{ml}$ in das nächste mit $9 \mathrm{ml}$ Bouillon aufgefüllte Reagenzglas pipettiert und auf dem Vortexer homogenisiert. Auf diese Weise entstand eine $1: 10$ Verdünnung, $10^{6} \mathrm{KbE} / \mathrm{ml}$ entsprechend.

- $100 \mu$ l aus dem zweiten Reagenzglas wurden dem nächsten (mit $10 \mathrm{ml}$ Bouillon) beigemengt. Nach Homogenisierung auf dem Vortexer lag eine $1: 1000$ Verdünnung, $10^{4} \mathrm{KbE} / \mathrm{ml}$ entsprechend, vor.

- aus dem dritten Reagenzglas wurde $1 \mathrm{ml}$ Bouillon in das nächste Reagenzglas (mit $9 \mathrm{ml}$ Bouillon) pipettiert. Nach Homogenisierung lag eine $1: 10000$ Verdünnung, $10^{3} \mathrm{KbE} / \mathrm{ml}$ entsprechend, vor.

$10^{3} \mathrm{KbE} / \mathrm{ml}$ erwiesen sich als zu gering für die Resistenztestung, sichtbar an nur sporadischem Sprosspilzwachstum, d. h. die Vertiefungen waren nicht bewachsen. Dieser Versuchsansatz war für eine MHK-Bestimmung nicht verwertbar.

Die geeignete Keimzahl für die MHK-Bestimmung lag bei $5 \times 10^{3}$ $\mathrm{KbE} / \mathrm{ml}$. Sie ergab sich durch das Mischen der zwei letzten Verdünnungsreihen im Verhältnis $1: 1$.

\section{Beimpfung der Platten \\ $\nabla$}

Die Mikrotestplatten mit vorgelegten und dehydratisierten Antimykotika wurden mit $100 \mu$ l Sprosszellsuspension pro Vertiefung manuell mit der Achtkanalpipette (Mikronaut Pipette) oder automatisiert mit dem Mikronaut Sprint beimpft. Die automatisierte Beimpfung ließ sich trotz vieler Vorteile (u. a. niedriges Kontaminationsrisiko) nur schwer durchführen. Die lipidhaltige Bouillon schäumte, was die Genauigkeit der Testung beeinträchtigte, so dass auf manuelles Pipettieren zurückgegriffen wurde. In der letzten Reihe lag die Wachstumskontrolle. Sie wurde mit Sprosszellbouillon aufgefüllt. Die Ergebnisse durften erst dann abgelesen werden, wenn Wachstum in der Kontrollvertiefung sichtbar wurde, hier nach vier Tagen.

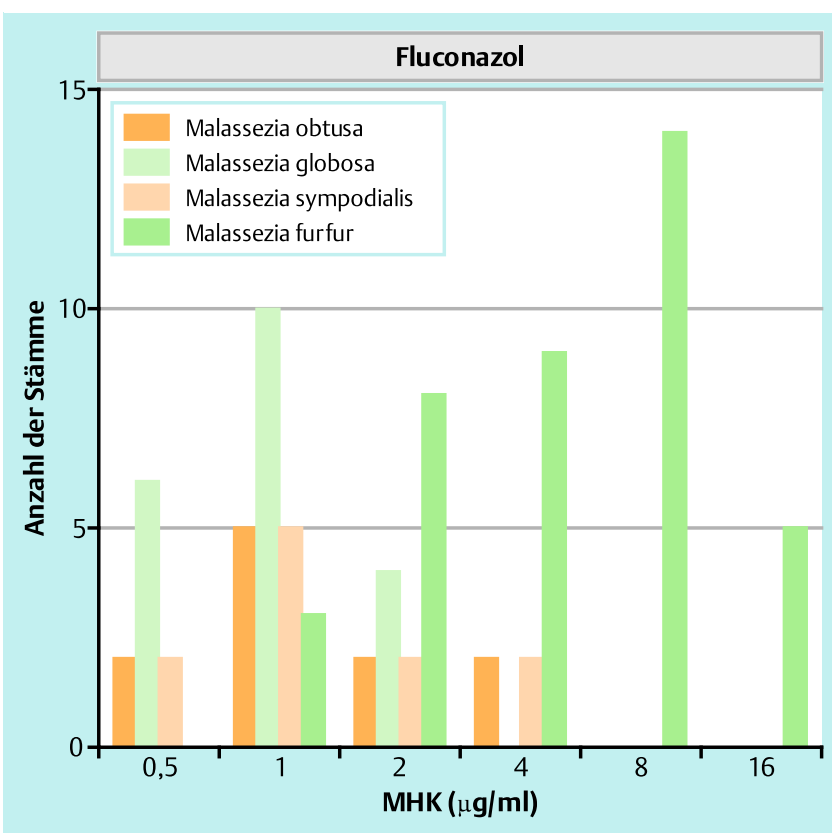

Abb. 1 Minimale Hemmkonzentrationswerte von Fluconazol gegenüber Malassezia. Mikrodilutionstest mit mDixon-Medium, Patientenisolate sowie Referenzstämme von Malassezia (insgesamt $\mathrm{n}=81$ Stämme): $M$. obtusa $\mathrm{n}=11$ Stämme, . globosa $\mathrm{n}=20$, M. sympodialis $\mathrm{n}=11$, M. furfur $\mathrm{n}=39$.

Nach Beimpfung wurden die Platten mit Parafilm verschlossen, damit die Bouillon nicht austrocknete und vor allem nicht kontaminiert werden konnten. Die MHK ist als die niedrigste Konzentration eines Antimykotikums, bei der kein Wachstum festgestellt werden kann (Endpunkt), definiert.

\section{Ergebnisse}

In-vitro-Empfindlichkeit von Malassezia-Wildstämmen Die MHK-Werte der einzelnen Malassezia-Spezies variierten innerhalb eines Antimykotikums. Der Vergleich zwischen den eingesetzten Wirkstoffen erbrachte Unterschiede. Wie erwartet, hemmten die Azolantimykotika (Fluconazol, Ketoconazol, Voriconazol und Itraconazol) das Wachstum in vitro schon bei niedrigen Konzentrationen.

Fluconazol rangierte bei Hemmkonzentrationen von $0,5 \mu \mathrm{g} / \mathrm{ml}$ bis $16 \mu \mathrm{g} / \mathrm{ml}$ (geometrisches Mittel 2,0) ( $\bullet$ Abb. 1). Ketoconazol zeigte eine sehr gute In vitro-Aktivität mit Werten von 0,03 bis $0,5 \mu \mathrm{g} / \mathrm{ml}$ (geometrisches Mittel 0,06), noch niedrigere Werte wurden mit dem Voriconazol (von 0,007 bis $1 \mu \mathrm{g} / \mathrm{ml}$ ) und Itraconazol $(0,007$ bis $0,5 \mu \mathrm{g} / \mathrm{ml})$ erreicht ( $\bullet$ Abb. $2-4)$. Bemerkenswert war, dass $M$. furfur im Vergleich zu anderen Spezies erst bei höheren Antimykotikakonzentrationen gehemmt wurde, was statistisch signifikant war $(\mathrm{p}<0,05)$.

5 -Flucytosin war in wenigen Fällen in der Lage, das Wachstum von Malassezia in vitro zu hemmen, es waren mit Werten zwischen 0,5 und $64 \mu \mathrm{g} / \mathrm{ml}$ sehr hohe Konzentrationen erforderlich (॰ Abb. 5).

Die Verteilung der MHK-Werte für Amphotericin B (Polyenantimykotikum) weist auf eine gute In-vitro-Wachstumshemmung hin, die Werte lagen zwischen 0,03125 und $8 \mu \mathrm{g} / \mathrm{ml}$ ( $\bullet$ Abb. 6). 


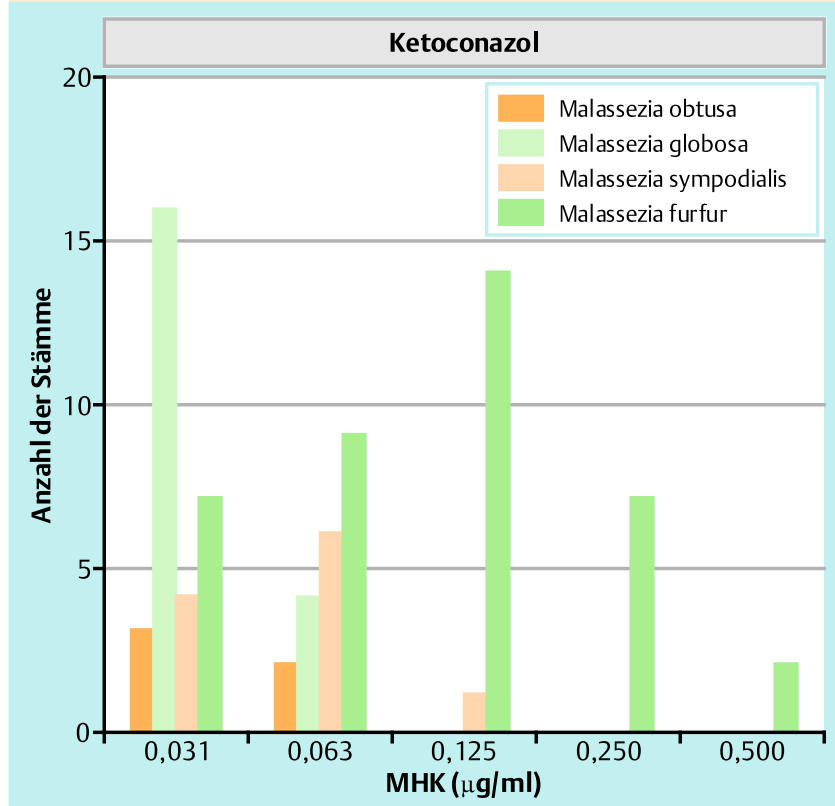

Abb. 2 Minimale Hemmkonzentrationswerte von Ketoconazol gegenüber Malassezia. Mikrodilutionstest mit mDixon-Medium, Patientenisolate sowie Referenzstämme von Malassezia (insgesamt $n=75$ Stämme): $M$. obtusa $\mathrm{n}=5$ Stämme, $M$. globosa $\mathrm{n}=20$, M. sympodialis $\mathrm{n}=11$, $M$. furfur $\mathrm{n}=39$.

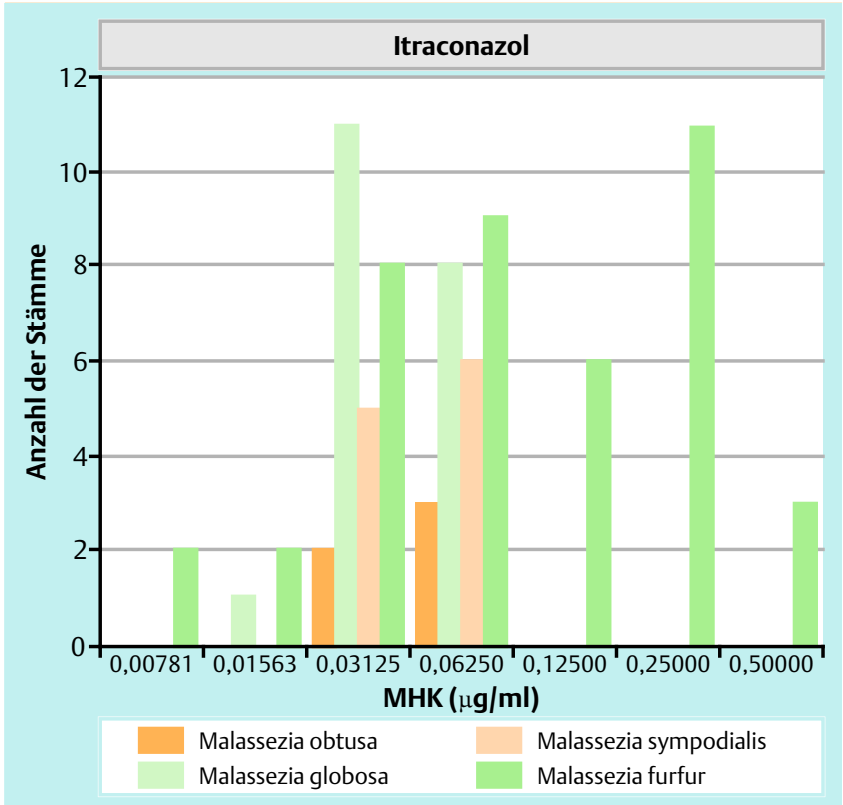

Abb. 4 Minimale Hemmkonzentrationswerte von Itraconazol gegenüber Malassezia. Mikrodilutionstest mit mDixon-Medium, Patientenisolate sowie Referenzstämme von Malassezia (insgesamt $n=75$ Stämme): $M$. obtusa $\mathrm{n}=5$ Stämme, $M$. globosa $\mathrm{n}=20$, M. sympodialis $\mathrm{n}=11, M$. furfur $\mathrm{n}=39$.

\section{In-vitro-Empfindlichkeit von Malassezia-Referenz- stämmen}

Die M. furfur-Referenzstämme (CBS 1878, CBS 6000, CBS 4162, CBS 6046, CBS 4170, Leiden, EG 658) erwiesen sich im Vergleich zu den $M$. furfur-Wildisolaten als weniger empfindlich gegen Fluconazol, Voriconazol und Itraconazol. Die MHK-Werte von Fluconazol rangierten bei Hemmkonzentrationen von $8-16 \mu \mathrm{g} /$ $\mathrm{ml}$ (geometrisches Mittel $8 \mu \mathrm{g} / \mathrm{ml}$ ). Die Werte für Voriconazol la-

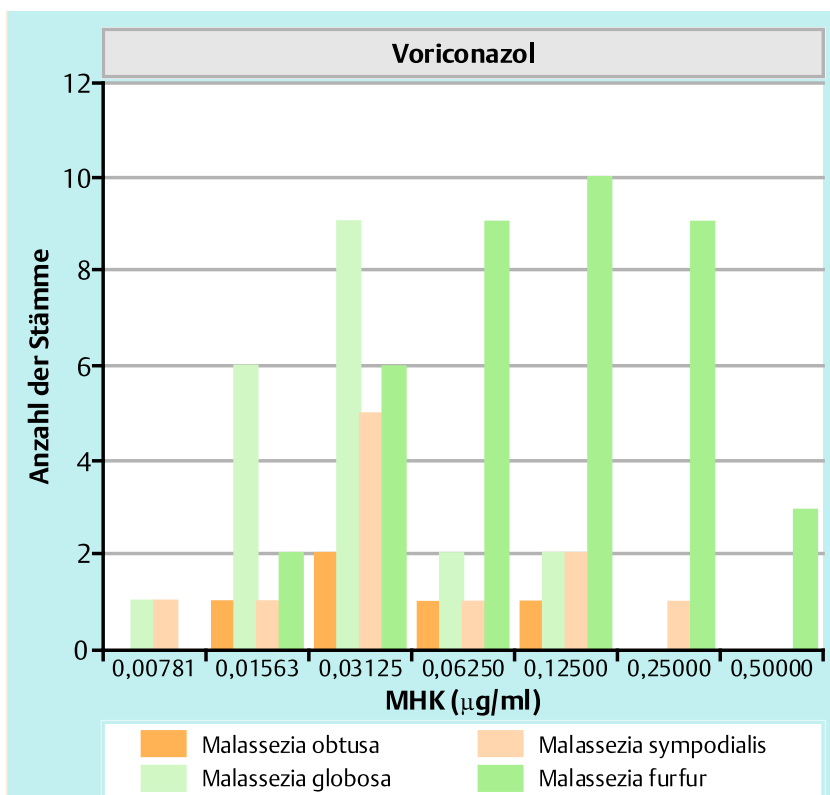

Abb. 3 Minimale Hemmkonzentrationswerte von Voriconazol gegenüber Malassezia. Mikrodilutionstest mit mDixon-Medium, Patientenisolate sowie Referenzstämme von Malassezia (insgesamt $n=75$ Stämme): $M$. obtusa $\mathrm{n}=5$ Stämme, $M$. globosa $\mathrm{n}=20, M$. sympodialis $\mathrm{n}=11, M$. furfur $\mathrm{n}=39$.

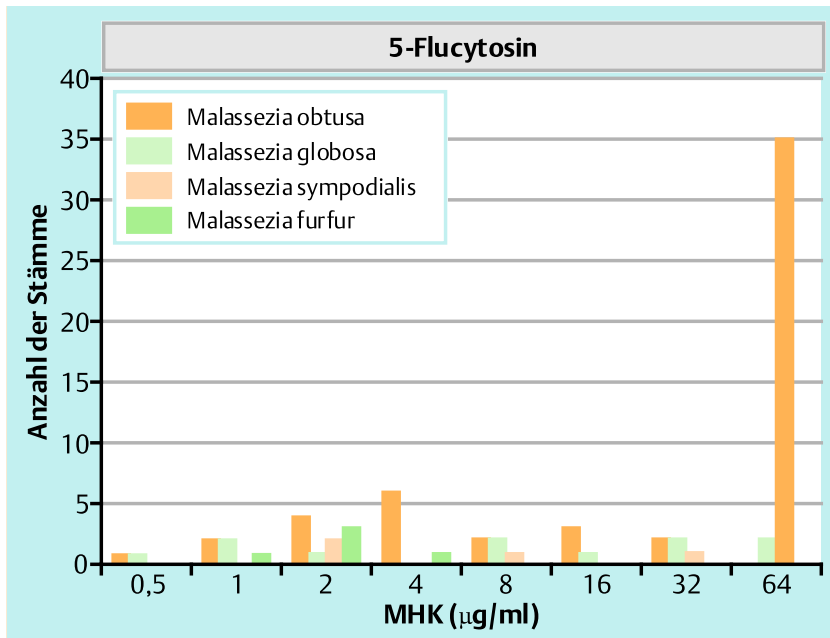

Abb. 5 Minimale Hemmkonzentrationswerte von 5-Flucytosin gegenüber Malassezia. Mikrodilutionstest mit mDixon-Medium, Patientenisolate sowie Referenzstämme von Malassezia (insgesamt $n=75$ Stämme): $M$. $o b$ tusa $\mathrm{n}=5$ Stämme, $M$. globosa $\mathrm{n}=20$, M. sympodialis $\mathrm{n}=11$, . furfur $\mathrm{n}=39$.

gen bei Hemmkonzentrationen von $0,06-0,5 \mu \mathrm{g} / \mathrm{ml}$ (geometrisches Mittel 0,25 $\mu \mathrm{g} / \mathrm{ml}$ ). Der Bereich der MHK-Werte für Itraconazol erstreckte sich von 0,06-0,25 $\mu \mathrm{g} / \mathrm{ml}$ (geometrisches Mittel 0,25). Die Hemmkonzentrationen derselben Antimykotika gegenüber den Malassezia-Wildstämmen betrugen jeweils für Fluconazol $1-16 \mu \mathrm{g} / \mathrm{ml}$ (geometrisches Mittel $4,0 \mu \mathrm{g} / \mathrm{ml}$ ), für Voriconazol $0,01-0,5 \mu \mathrm{g} / \mathrm{ml}$ (geometrisches Mittel $0,125 \mu \mathrm{g} / \mathrm{ml}$ ) und für Itraconazol 0,007-0,5 $\mu \mathrm{g} / \mathrm{ml}$ (geometrisches Mittel 0,06).

Die unterschiedlichen MHK-Werte für Referenz- und Wildstämme von M. furfur sind statistisch signifikant $(p<0,05)$. Um die Wachstumshemmung zu erreichen, waren höhere Konzentra- 


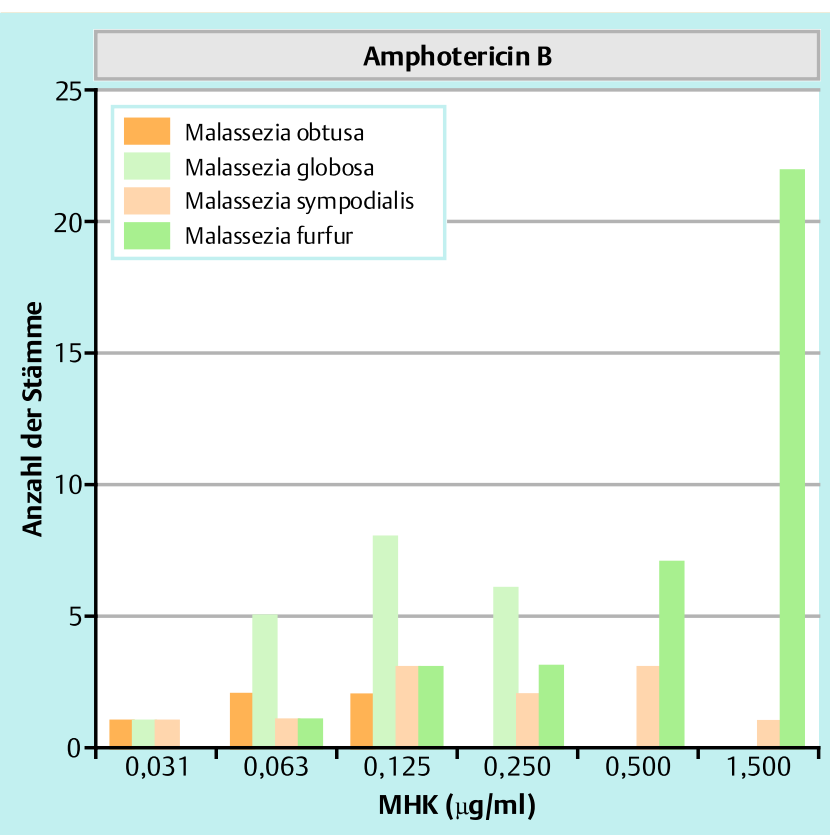

Abb. 6 Minimale Hemmkonzentrationswerte von Amphotericin B gegenüber Malassezia. Mikrodilutionstest mit mDixon-Medium, Patientenisolate sowie Referenzstämme von Malassezia (insgesamt $\mathrm{n}=75$ Stämme): M. obtusa $\mathrm{n}=5$ Stämme, $M$. globosa $\mathrm{n}=20$, M. sympodialis $\mathrm{n}=11$, M. furfur $\mathrm{n}=39$.

tionen von Antimykotika bei M. furfur-Referenzstämmen erforderlich, was ebenfalls statistisch signifikant war.

\section{Diskussion \\ $\nabla$}

Antimykotika, an erster Stelle das Azolderivat Ketoconazol, werden erfolgreich zur lokalen Behandlung der Malassezia-assoziierten Dermatosen eingesetzt [23]. Darüber hinaus finden weitere systemisch applizierbare Triazole, vor allem Itraconazol und Fluconazol, Anwendung zur Therapie ausgeprägter und rezidivierender Malassezia-Infektionen, insbesondere der Pityriasis versicolor.

Hier stand im Fokus der Untersuchungen, festzustellen, ob es Malassezia-Isolate, Wild- und Referenzstämme, gibt, die in vitro eine verminderte Empfindlichkeit, ggf. eine In-vitro-Resistenz, gegenüber Antimykotika aufweisen.

Eine bewährte Methode zur Empfindlichkeitstestung von antimykotisch wirkenden Substanzen ist der Agardilutionstest $[24,25]$. Darüber hinaus wurde eine Mikrodilutionstechnik zur Empfindlichkeitstestung von Malassezia verwendet [26]. Die Vorkultivierung der $M$. furfur-Stämme sowie die Empfindlichkeitstestung erfolgen dabei im Leeming-Notman-Medium [20]. Wegen der starken Hintergrundtrübung dieses Mediums wird die Wachstumshemmung mittels einer kolorimetrischen Detektion der metabolischen Aktivität bestimmt. Als Indikator der metabolischen Aktivität ist dem Medium der Oxidations-/Reduktionsindikator AlamarBlue ${ }^{\mathrm{TM}}$ zugesetzt, so dass die Farbe der Lösung blau ist. Der MHK-Wert ist die niedrigste Konzentration, bei der eine blaue oder Blau-rosa-Färbung noch erkennbar war. Wachstum erkennt man an der rosa-roten Färbung.

Eine neuere Methode zur Empfindlichkeitstestung von Malassezia basiert auf Christensen-Harnstoff-Medium, welchem eine Lipidquelle in Form von Tween 40 und 80 zugesetzt wird. Wachs- tum äußert sich in der roten Färbung des Mediums, die MHKWerte bestimmt man als die niedrigste Wirkstoffkonzentration, bei der die rote Färbung nicht erkennbar ist [27].

Eine gebräuchliche Methode der In-vitro-Empfindlichkeitstestungen von Malassezia ist die hier angewandte Bestimmung der minimalen Hemmkonzentration mittels Rehydratisierung von Antimykotika durch die Zugabe einer standardisierten Hefezellsuspension in die Antimykotika-haltigen Vertiefungen der Mikrotitrationsplatten. Velegraki et al. [28] verwendeten ein mit Fettsäuren supplementiertes RPMI 1640-Medium, welches sonst ohne Fettzusatz entsprechend NCCLS-Methode zur Empfindlichkeitstestung von Candida-Arten verwendet wird.

Problematisch bei der Empfindlichkeitstestung des lipophilen Hefepilzes Malassezia sind die oft zu Klumpen aggregierten Sprosszellen, welche schwer in einer wässrigen Lösung zu suspendieren sind, dementsprechend ist die Einstellung der Keimdichte für das Inokulum nur eingeschränkt möglich. Durch die hier verwendete modifizierte Dixon-Bouillon (mDixon-Bouillon ohne Pepton) konnte das Problem gelöst werden, eine genaue Einstellung der Keimdichte war möglich.

In der Bouillon waren keine Substanzen mit einer antagonistischen Wirkung gegenüber Antimykotika enthalten. Im Gegensatz dazu sind bivalente Metallionen $\left(\mathrm{Ca}^{2+}, \mathrm{Mg}^{2+}\right)$ Bestandteil synthetischer Medien. Diese Ionen konkurrieren mit den Imidazolen (Ketoconazol) um die Bindungsplätze und reduzieren so deren effektive Konzentration [29]. Die Subkultivierung aller Stämme fand auf dem lipidhaltigen Nährmedium statt. Die kulturelle Anzucht und Empfindlichkeitstestung wurde bei der Temperatur von $37^{\circ} \mathrm{C}$ durchgeführt. Prinzipiell möglich ist auch eine niedrigere Temperatur von ca. $30^{\circ} \mathrm{C}$.

Neben Azolantimykotika - Fluconazol, Itraconazol, Ketoconazol und Voriconazol - wurde die Wirkung des Polyenantimykotikums Amphotericin B und von 5-Flucytosin auf das Wachstum von Malassezia untersucht. Hervorzuheben ist, dass die hier gefundenen MHK-Werte mit anderen Studien übereinstimmen $[19,26,30$ - 34]. Sie lagen unter den - jedoch für Candida-Spezies - angegebenen NCCLS-Grenzwerten, d.h., eine In-vitro-Resistenz bestand nicht. Es sei jedoch nochmals betont, dass eine verbindliche Standardisierung der Labormethoden zur In-vitroEmpfindlichkeitstestung von Hefen der Gattung Malassezia nicht vorliegt.

Die MHK-Werte der einzelnen Malassezia-Stämme variierten innerhalb eines Antimykotikums. Der Vergleich zwischen den Wirkstoffen erbrachte erhebliche Unterschiede. Es fand sich ein hervorragender Hemmeffekt der Azolantimykotika gegen $\mathrm{Ma}$ lassezia. Voriconazol, Itraconazol und Ketoconazol hemmten das Wachstum in vitro am stärksten, was mit anderen Studien übereinstimmt [7,19,35-38]. Voriconazol hemmte schon in sehr niedrigen Konzentrationen das Wachstum von Malassezia [19]. Die M. furfur-Referenzstämme erwiesen sich im Vergleich zu den Wildisolaten als weniger empfindlich gegen Fluconazol, Voriconazol und Itraconazol.

Aktuelle Arbeiten bestätigen, dass es unter individuellen Malassezia-Spezies Unterschiede der In-vitro-Empfindlichkeit gibt. $M$. furfur ist am wenigsten empfindlich gegenüber Antimykotika (Ketoconazol, Econazol, Miconazol) und sogar Teebaumöl, dagegen sind M. sympodialis, M. slooffiae, M. globosa sowie M. obtusa deutlich empfindlicher. Das findet eine sehr gute Entsprechung in der vorliegenden Untersuchung. Malassezia furfur ist am wenigsten empfindlich gegenüber mehreren Antimykotika, dagegen sind M. sympodialis, M. globosa und M. obtusa deutlich empfindlicher, d. h. sie wiesen niedrigere MHK-Werte auf. Es wird die 
Notwendigkeit betont, dass für eine adäquate antimykotische Therapie die Kenntnis der M.-Spezies Voraussetzung ist [32].

Das Allylaminantimykotikum Terbinafin wies hier sehr unterschiedliche MHK-Werte auf.

Die Werte für M. furfur schwankten von deutlich empfindlichen bis zu in vitro scheinbar resistenten Stämmen. Bemerkenswert ist, dass orales Terbinafin heute auch schon beim atopischen und beim seborrhoischen Ekzem in Gesicht mit Erfolg eingesetzt wird. Möglicherweise beruht der Effekt auf einer Reduktion von Malassezia-Antigen in den Läsionen $[39,40]$.

Die Empfindlichkeitsunterschiede der einzelnen Spezies stimmen mit denen aus aktuellen Studien überein. Die Werte lagen jedoch in niedrigeren Bereichen, was auf die Messung der metabolischen Aktivität und nicht wie bei den anderen Methoden auf die Zellzahl zurückzuführen ist [41].

Das Polyenantimykotikum Amphotericin B wies auf eine gute Wachstumshemmung in vitro hin. Dieses seit Jahrzehnten bekannte Antimykotikum besitzt ein breites Wirkungsspektrum, zeichnet sich jedoch durch hohe Myelo-, Hepato- und vor allem Nephrotoxizität aus.

Dagegen war 5-Flucytosin in wenigen Fällen in der Lage, das Wachstum von Malassezia zu hemmen, zumindest waren sehr hohe Konzentrationen erforderlich. 5-Flucytosin ist ein ausschließlich systemisch einsetzbares Antimykotikum zur paraenteralen Behandlung invasiver Mykosen. Hier wurde 5-Flucytosin untersucht, um herauszufinden, ob ein möglicher Einsatz bei den oben beschriebenen Malassezia-Fungämien denkbar wäre. Dem scheint nicht so, sichtbar an den in vitro vergleichsweise hohen MHK-Werten.

Obwohl die Malassezia-Spezies differierende antimykotische Empfindlichkeiten in vitro aufweisen, muss die Effizienz der Antimykotika, d.h. die Korrelation von In-vitro-MHK-Werten mit dem klinischen Ansprechen der antimykotischen Therapie in vivo, zukünftigen Untersuchungen vorbehalten sein. Überdies kann die Verabreichungsform - topisch vs. oral - die Therapie und Effizienz des Mittels beeinflussen [42 - 44].

\section{Fazit fïr die Praxis}

$\nabla$

Die in dieser Arbeit angewandte Methode der In-vitro-Empfindlichkeitstestung mittels Mikrodilutionstechnik lieferte niedrige und plausible MHK-Werte für die modernen Azolantimykotika gegenüber dem lipophilen Hefepilz Malassezia spp. Offensichtlich gibt es Unterschiede der In-vitro-Empfindlichkeit verschiedener Malassezia-Arten. M. furfur ist am wenigsten empfindlich gegenüber mehreren Antimykotika, während M. sympodialis, $M$. globosa und $M$. obtusa deutlich empfindlicher sind, d. h. bereits bei niedrigeren Werten im Wachstum gehemmt wurden. Noch nicht geklärt ist jedoch, ob es eine Korrelation der In-vitro-Aktivität zur antimykotischen Therapie der Malassezia-assoziierten Dermatosen gibt.

\section{Abstract}

\section{Species Specific Susceptibility of Malassezia against Antifungal Agents in vitro \\ $\nabla$}

Yeasts of the genus Malassezia are part of the normal flora of human skin. Under appropriate conditions they may cause skin infections such as pityriasis versicolor, Malassezia folliculitis, se- borrhoeic dermatitis, and dandruff. It has been reported that Malassezia may be a trigger factor for atopic dermatitis.

The in vitro antifungal susceptibility testing of Malassezia still remains a problem. There is no recommended method of antifungal susceptibility testing designed for lipophilic genus Malassezia.

81 strains of Malassezia were examined for their in vitro susceptibility to antifungal substances ( 17 were reference or pre-identified strains, 64 were obtained from clinical specimens). Identification of the clinical strains based on biochemical features and Fourier-Transform-Infrared-Spectroscopy. All strains were maintained 7 days on modified Dixon agar. In vitro susceptibility testing was performed on microtitration plates with laid out of 6 dehydrated antifungals (ketoconazole, voriconazole, itraconazole, fluconazole, amphotericin B, and 5-flucytosin) in 12 different concentrations. The yeast cell inoculum was adjusted to $5 \times 10^{3}$ cfu (colony forming unit) suspended in modified Dixon without peptone.

All Malassezia strains were very susceptible to the azole drugs. Especially, voriconazole (MIC - minimum inhibitory concentration - 0.007-1 $\mu \mathrm{g} / \mathrm{ml}$ ), itraconazole (MIC $0.007-0.05 \mu \mathrm{g} / \mathrm{ml}$ ), and ketoconazole (MIC $0.03-0.5 \mu \mathrm{g} / \mathrm{ml}$ ) were the most effective agents. MIC values among Malassezia sympodialis, Malassezia globosa and Malassezia obtusa were comparable, whereas MIC values for $M$. furfur were significantly higher $(\mathrm{p}<0.05)$. All values ranged among those accepted according the NCCLS standards.

Amphotericin B was also effective against Malassezia in vitro (MIC $0.03125-8 \mu \mathrm{g} / \mathrm{ml}$ ). 5-Flucytosin was unlikely to inhibit the growth of Malassezia. In some cases it could prevent the growth, however high concentrations were needed.

There were variations in susceptibility of different Malassezia species to azole antifungal substances. Malassezia furfur was found to be the less susceptible species when compared to $\mathrm{Ma}$ lassezia obtusa, Malassezia globosa and Malassezia sympodialis.

\section{Literatur}

1 Marcon MJ, Powell DA. Human infections due to Malassezia spp. Clin Microbiol Rev 1992; 5: 101 - 109

2 Zomorodian K, Tarazooei B, Kordbacheh P, Zaini F, Zeraati H, Saadat F, Hallaji Z, Mirhendi SH, Geramishoar M. Isolation and identification of Malassezia spp. in seborrhoeic dermatitis and healthy skin. Mikol Lek 2004; 11 (Suppl 1): 68 (abstract)

3 Nenoff $P$. Malassezia als Opportunist und Protagonist. Malassezia und seborrhoisches Ekzem: kausale oder zufällige Assoziation? ÄP Ärztliche Praxis Dermatologie 2004; 2: 33-34

4 Crespo Erchiga V, Ojedo Martos A, Vera Casano A, Crespo Erchiga A, Sanchez Fajardo F, Guého E. Mycology of pityriasis versicolor. J Mycol Med 1999; 9: $143-148$

5 Ashbee HR. Recent developments in the immunology and biology of Malassezia species. FEMS Immunol Med Microbiol 2006; 47: 14-23

6 Crespo-Erchiga V, Florencio VD. Malassezia yeasts and pityriasis versicolor. Curr Opin Infect Dis 2006; 19: 139-147

7 Nenoff $P$, Reinl P, Haustein U-F. Malassezia: Erreger, Pathogenese und Therapie. Hautarzt 2001; 52: 73-86

8 Faergemann J. The role of the Malassezia yeast in skin diseases. Mikol Lek 2004; 11: 129-132

9 DeAngelis Y, Leland MO, Boekhout T et al. Three etiologic facets of dandruff and seborrheic dermatitis: Malassezia fungi, sebaceous lipids, and individual sensitivity. Poster abstract P 10.132 of the 4th International Meeting of Hair Research Societies, June 17-19, 2004, Berlin, JDDG 2: 550 (abstract) 2004

10 DeAngelis YM, Gemmer CM, Kaczvinsky JR, Kenneally DC, Schwartz JR, Dawson TL Jr. Three etiologic facets of dandruff and seborrheic dermatitis: Malassezia fungi, sebaceous lipids, and individual sensitivity. J Investig Dermatol Symp Proc 2005; 10: 295-297 
11 Scherdin U, Rippke F. Kopfschuppen - Pathogenese und Wirksamkeit eines neuen Anti-Schuppen Shampoos. Derm Praktische Dermatologie 2004; 10: $265-267$

12 Guillot J, Guého E, Lesourd M, Midgley G, Chevrier G. Identification of Malassezia yeasts: a practical approach. J Mycol Med 1996; 6: 103 110

13 Romano C, Ghilardi A, Nardoni S, Mancianti F. Species of Malassezia and chronic forms of pityriasis versicolor. Mikol Lek 2004; 11 (Suppl 1): 67 (abstract)

14 Sugita T, Takashima M, Shinoda T, Suto H, Unno T, Tsuboi R, Ogawa H, Nishikawa A. New yeast species, Malassezia dermatis, isolated from patients with atopic dermatitis. J Clin Microbiol 2002; 40: 1363-1367

15 Sugita T, Takashima M, Kodama M, Tsuboi R, Nishikawa A. Description of a new yeast species, Malassezia japonica, and its detection in patients with atopic dermatitis and healthy subjects. J Clin Microbiol 2003; 41: 4695-4699

16 Gupta AK, Boekhout T, Theelen B, Summerbell R, Batra R. Identification and typing of Malassezia species by amplified fragment length polymorphism and sequence analyses of the internal transcribed spacer and large-subunit regions of ribosomal DNA. J Clin Microbiol 2004; 42: $4253-4260$

17 Nenoff $P$. In vitro-Empfindlichkeitstestung von Malassezia furfur. Zeitschr H+G 1997; 72: 104- 109

18 Nenoff $P$, Haustein UF. In vitro susceptibility testing of Malassezia furfur against rilopirox. Skin Pharmacol 1997; 10: 275-280

19 Gupta AK, Kohli Y, Li A, Faergemann J, Summerbell RC. In vitro susceptibility of the seven Malassezia species to ketoconazole, voriconazole, itraconazole and terbinafine. Br J Dermatol. 2000; 142: 758 - 765

20 Leeming JP, Notmann FH. Improved methods for isolation and enumeration of Malassezia furfur from human skin. J Clin Microbiol 1987; 25: 2017-2019

21 Kurtzman CP, Fell JW. The Yeasts, the taxonomic study. 4th edn. Amsterdam: Elsevier Science Publisher B. V., 2000

22 Kalinowska-Pujdak A, Schmalreck A, Haustein UF, Nenoff P. SpeziesDifferenzierung von Hefen der Gattung Malassezia mittels FourierTransform-Infrarot-Spektroskopie. Hautarzt 2006; 57: 127-136

23 Thoma W, Mayser P. Malassezia-Hefen und ihre Bedeutung. Dt Dermatol 2004; 52: 260 - 265

24 Nenoff $P$, Haustein UF. In vitro activitiy of phytosphingosines against Malassezia furfur and Candida albicans. Acta Dermato-Venereol 2002; 82: $170-173$

25 Faergemann J, Ausma J, Borgers M. In vitro activity of R126638 and ketoconazole against Malassezia species. Acta Derm Venereol 2006; 86: $312-315$

26 Schmidt A, Rühl-Hörster B. In vitro susceptibility of Malassezia furfur against azole compounds. Mycoses 1996; 39: 309-312

27 Murai T, Nakamura Y, Kano R, Watanabe S, Hasegawa A. Susceptibility testing of Malassezia pachydermatis using the urea broth microdilution method. Mycoses 2002; 45: 84-87

28 Velegraki A, Alexopoulos EC, Kritikou S, Gaitanis G. Use of fatty acid RPMI 1640 media for testing susceptibilities of eight Malassezia species to the new triazole posaconazole and to six established antifungal agents by a modified NCCLS M27-A2 microdilution method and Etest. J Clin Microbiol 2004; 42: 3589- 3593
29 Swamy KHS, Sirsi M, Rao GR. Studies on the mechanism of action of miconazole on respiration and cell permeability of Candida albicans. Antimicrob Agents Chemother 1974; 5: 420-425

30 Nenoff $P$, Haustein $U$-F. In vitro susceptibility testing of Pityrosporum ovale against antifugal, antiseborrheic and antipsoriatic agents. J Eur Acad Dermatol Venereol 1994; 3: 331 - 333

31 Nenoff $P$, Haustein U-F. Antifungal activity of the essential oil of Melaleuca alternifolia (tea tree oil) against pathogenic fungi in vitro. Skin Pharmacol 1996; 9: 388-394

32 Hammer KA, Carson CF, Riley TV. In vitro activities of ketoconazole, econazole, miconazole and Melaleuca alternifolia (tea tree) oil against Malassezia species. Antimicrob Agents Chemother 2000; 44: 467 469

33 Van Gerven F, Odds FC. The anti-Malassezia furfur activity in vitro and in experimental dermatitis of six imidazole antifungal agents: bifonazole, clotrimazole, flutrimazole, ketoconazole, miconazole and sertaconazole. Mycoses 1995; 38: 389- 393

34 Faergemann J, Borgers $M$. The effect of ketoconazole and itraconazole on the filamentous form of Pityrosporum ovale. Acta Derm Venereol 1990; 70: $172-176$

35 Faergemann J. Treatment of pityriasis versicolor with single dose of fluconazole. Acta Derm Venereol 1992; 72: 74 - 75

36 Strippoli V, Piacentini A, D'Auria FD, Simonetti N. Antifungal activity of ketoconazole and other azoles against Malassezia furfur in vitro and in vivo. Infection 1997; 25: 303-306

37 Garau M, Pereiro M Jr, del Palacio A. In vitro susceptibilities of Malassezia species to a new triazole, albaconazole (UR-9825), and other antifungal compounds. Antimicrob Agents Chemother 2003; 47: 2342 2344

38 Sancak B, Ayhan M, Karaduman A, Arikan S. In vitro activity of ketoconazole, itraconazole and terbinafine against Malassezia strains isolated from neonates. Mikrobiyol Bul. 2005; 39: 301 - 308

39 Maejima H, Tokunaga C, Kaneko S, Mukai H, Abe A. Analysis of facial lesions on adult type atopic dermatitis with anti-fungus drug (terbinafine hydrochloride) - analysis of serum anti-Malassezia IgE antibody titers and histamine release test (Article in Japanese). Arerugi 2004; 53: $515-521$

40 Vena GA, Micali G, Santoianni P, Cassano N, Peruzzi E. Oral terbinafine in the treatment of multi-site seborrhoic dermatitis: a multicenter, double-blind placebo-controlled study. Int J Immunopathol Pharmacol 2005; 18 : $745-753$

41 Nakamura Y, Kano R, Murai T, Watanabe S, Hasegawa A. Susceptibility testing of Malassezia species using the urea broth microdilution method. Antimicrob Agents Chemother 2000; 44: 2185 - 2186

42 Kagawa S. Clinical efficiacy of terbinafine in 629 Japanese patients with dermatomycosis. Clin Exp Dermatol 1989; 14: 114- 115

43 Villars V, Jones TC. Present status of the efficiacy and tolerability of terbinafine (Lamisil) used systemically in the treatment of dermatomycoses of the skin. J Dermatol Treat 1990; 1: $33-38$

44 Aste N, Pau M, Pinna AL, Colombo MD, Biggio P. Clinical efficiacy and tolerability of terbinafine in patients with pityriasis versicolor. Mycoses $1991 ; 34: 353-357$ 